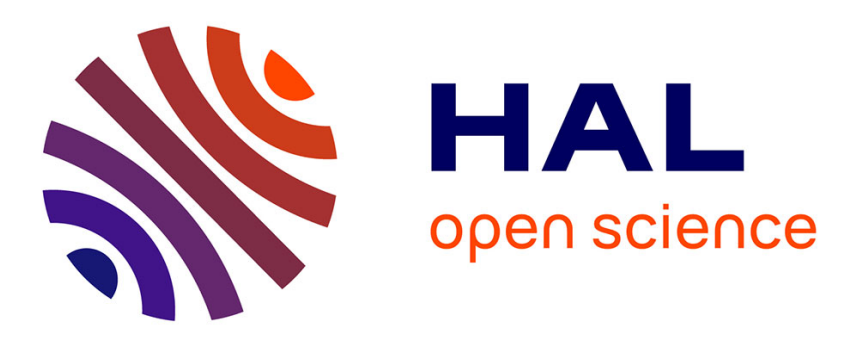

\title{
Derivation of supersaturation and nucleation flux during precipitation from the mixing pattern of an inert tracer in the same device: case of unmixed feed streams
}

R David, A Fall, Olivier Lecoq

\section{- To cite this version:}

R David, A Fall, Olivier Lecoq. Derivation of supersaturation and nucleation flux during precipitation from the mixing pattern of an inert tracer in the same device: case of unmixed feed streams. Chemical Engineering Science, 2003, 58 (13), pp.2883-2891. 10.1016/S0009-2509(03)00140-4 . hal-01717491

\author{
HAL Id: hal-01717491 \\ https://hal.science/hal-01717491
}

Submitted on 6 Nov 2019

HAL is a multi-disciplinary open access archive for the deposit and dissemination of scientific research documents, whether they are published or not. The documents may come from teaching and research institutions in France or abroad, or from public or private research centers.
L'archive ouverte pluridisciplinaire HAL, est destinée au dépôt et à la diffusion de documents scientifiques de niveau recherche, publiés ou non, émanant des établissements d'enseignement et de recherche français ou étrangers, des laboratoires publics ou privés. 


\title{
Derivation of supersaturation and nucleation flux during precipitation from the mixing pattern of an inert tracer in the same device: case of unmixed feed streams
}

\author{
René David*, Ahmed Fall, Olivier Lecoq \\ Laboratoire de Genie des Procédés des Solides Divisés, EMAC-CNRS, 81013 Albi Cedex 9, France
}

\begin{abstract}
Inert liquid tracer concentrations in a continuous mixer are analyzed by planar laser induced fluorescence (PLIF). Special attention is paid to the two separated entering feed streams containing the tracer solution or pure solvent. From the instantaneous tracer concentration fields, the method proposed allows one to easily calculate the instantaneous supersaturation fields, which would be obtained in the same mixing device with reagents instead of an inert tracer solution and pure solvent. A typical mixing situation in a stirred tank with separated feed streams is investigated. Maps of averaged supersaturation and averaged nucleation flux are yielded with high spatial resolution of a few tens of micrometers for each pixel. The method gives interesting indications about the ability of a given precipitator geometry to generate coarse or fine particles. However, it cannot be extended straightforward to partially premixed feed streams without the use of a mixing model.
\end{abstract}

Keywords: Mixing; Precipitation; PLIF; Nucleation; Tracer; Supersaturation

\section{Introduction}

Basically, precipitation is a chemical reaction between $\mathrm{A}$ and $\mathrm{B}$, yielding a non-soluble particulate precipitate $\mathrm{S}$ :

$n \mathrm{~A}+m \mathrm{~B} \rightarrow \mathrm{S}$ (solid),

where $n$ and $m$ generally lie between 1 and 3 .

Precipitation encompasses the so-called primary nucleation step, which generates the nuclei from the liquid phase and further steps forming the particle like growth, agglomeration, breakage or secondary nucleation, which generates additional nuclei from existing particles. Precipitations are generally characterized by small particles and short to medium precipitation times (Mersmann, 2001). According to the length and time scales defined by Villermaux and David (1988), this corresponds to high nucleation and medium growth rates.

Danckwerts (1952) first established the basic concepts and definitions of mixing characteristics of miscible liquids, i.e. segregation scale and intensity.

\footnotetext{
* Corresponding author. Fax: +33-563-49-3025.

E-mail address: rdavid@enstimac.fr (R. David)
}

The question of the relation between tracer experiments and extent of chemical reactions has been tackled by Levenspiel (1971, Chapter 10) several years ago. He demonstrated that the knowledge of the residence time distribution of an inert tracer was not sufficient to predict the local extent of a single or a set of chemical reactions, except for the case of a first-order reaction. Then, a micro-mixing model is needed.

In between, Shenoy and Toor (1990) showed that there is a relation between feed fraction of an inert tracer and the extent of an instantaneous chemical reaction, provided that the species have similar diffusivities.

The aim of the present paper is to demonstrate that, given simple assumptions, it is possible to predict the supersaturation and the nucleation flux fields in a mixed precipitator from concentration measurements of an inert tracer in the same precipitator geometry.

In Fig. 1 the different characteristic times of mixing and crystallization are reported. From Fig. 1, it results that, among the crystallization phenomena, only primary nucleation is faster than the final mixing steps in turbulent mixers. Primary nucleation begins as soon as the reacting species A and B are into contact. Other phenomena (crystal 


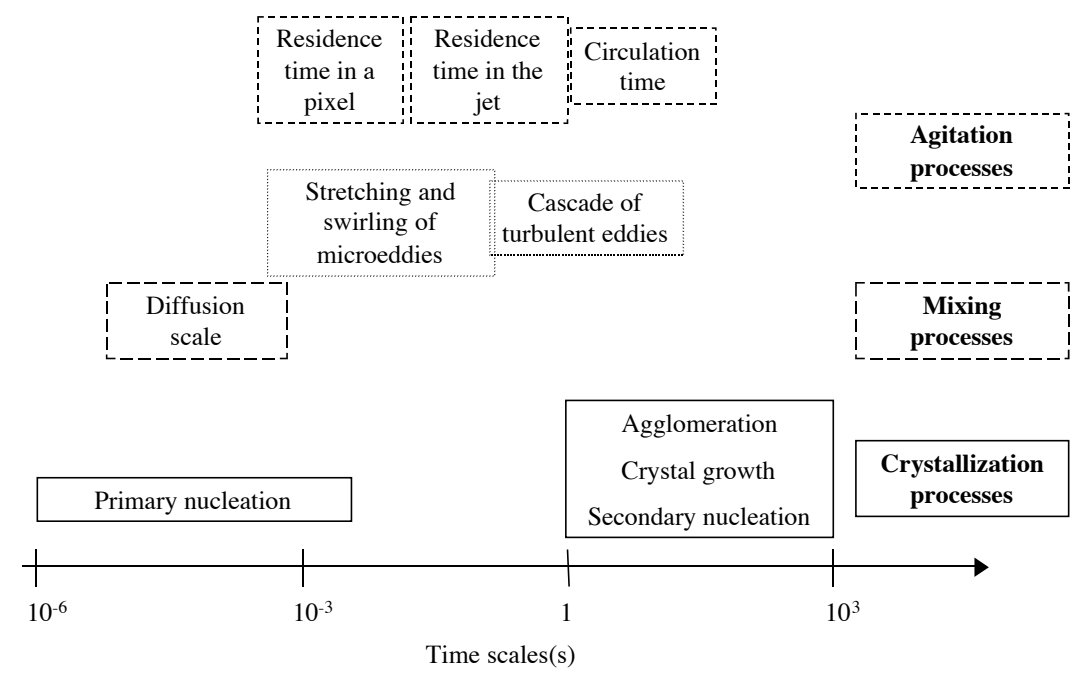

Fig. 1. Different characteristic time ranges of turbulent mixing and crystallization processes.

breakage, secondary nucleation, agglomeration) have characteristic times in the orders of magnitude of the internal circulation (i.e. macromixing) times. But, primary nucleation yields small nuclei. Therefore, it is not able to generate significant consumption of reagents during the very short nucleation times when it is not coupled with high growth rates.

\section{Experimental apparatus}

The simplified scheme of the apparatus used in this work (see Fall, Lecoq, \& David, 2001) is shown in Fig. 2. The experiments are carried out in a stirred tank of $20 \mathrm{dm}^{3}$ of standard configuration, with internal diameter $T=0.29 \mathrm{~m}$. The walls of the inner tank are equipped with four baffles of width $T / 10$ and thickness $T / 100$ at intervals of $90^{\circ}$. The impeller diameter (Rushton turbine) and bottom clearance are equal to $H / 3$, where $H=T$ represents the height of the liquid in the tank.

The stirring speed is set at $N=3.5 \mathrm{~s}^{-1}$. Two reservoirs continuously supply the tank with equal flow rates $\left(Q_{A}=\right.$ $Q_{B}=0.67 \mathrm{dm}^{3} \min ^{-1}$ ) by two incoming feed tubes (internal diameter $6 \mathrm{~mm}$ ). The two feed points are located at the tangential vertical plane to the impeller at $T / 10$ from the baffle; close to the fluid surface. The tank is operated in the continuous mode for tracer concentration measurements. The tracer is Rhodamine B.

The planar laser induced fluorescence technique (PLIF) combined with image processing, which is a powerful method to characterize the mixing in stirred tanks is used (Houcine, Vivier, Plasari, David, \& Villermaux, 1996; Fall et al., 2001). It consists of the measurement of the fluorescence intensity of a tracer excited by a very thin planar laser sheet. This intensity is transformed into a local instantaneous concentration field by a calibration procedure, (Houcine et al., 1996; Fall et al., 2001).
The mixing pattern is described in Figs. 3: Fig. 3a shows the mixing of the solution of the inert tracer, and Fig. $3 \mathrm{~b}$ the mixing of two reagents $\mathrm{A}$ and $\mathrm{B}$ in the same mixing device. Note that A-feed in the reactive case (tube on the right-hand side) corresponds to the tracer feed in the inert situation.

As both fluid flows are fed separately, each entering jet can be considered as mixing separately with its bulk environment. These jets are structured in three zones (Fig. 5):

- A conical core zone quoted 1 , where the concentration is very close to the feed concentration,

- A mixing zone 2, where the concentrations result from the mixing process, which is in turn governed by the velocity fields generated by the jet and the inner flow in the mixing device,

- And, finally, the bulk 3, where the concentrations are relatively uniform in space due to efficient mixing.

As stated above, the mixing process in the jets is rapid when compared with the circulation times. Therefore, only primary nucleation takes place and no significant consumption of reagents is likely to occur during the transition time of the fluid in the jets. This assumption will be discussed afterwards more in detail.

\section{Experimental results with an inert tracer}

The mixing device, which is tested here, is a standard tank equipped with a Rushton turbine. Tangentially to the envelope of the impeller, the measurement plane contains two opposite feed pipes, feeding either fresh tracer solution or pure solvent downwards.

An example of instantaneous reduced local concentration of the tracer $X_{T}$ is shown in Fig. 4 for the mixing device described above. It varies between 0 (black) in the pure 

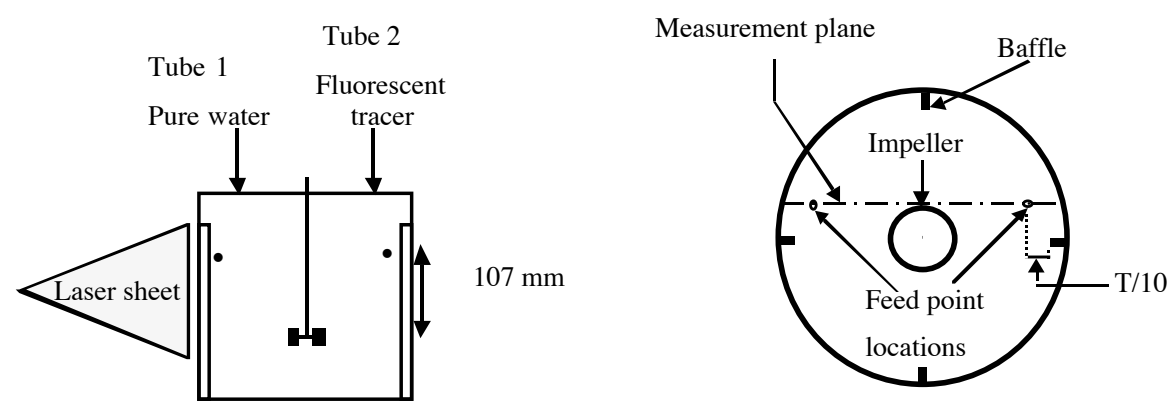

Fig. 2. Experimental set-up.
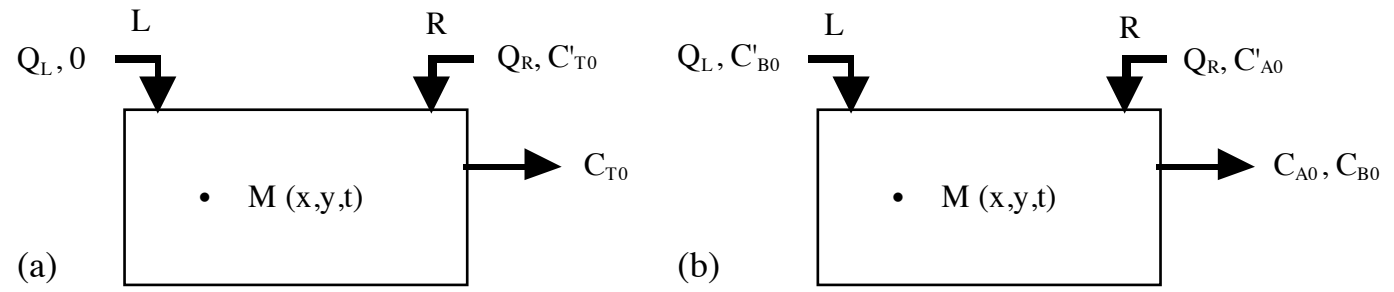

Fig. 3. The mixing pattern: (a) The mixing of the solution of inert tracer and (b) The mixing of two reagents A and B.

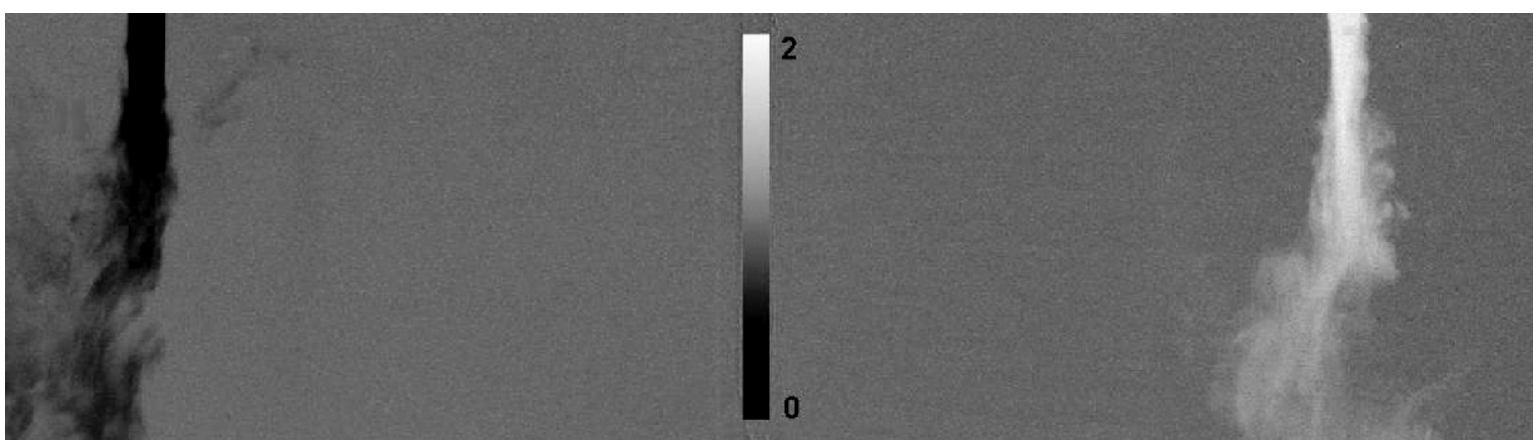

Fig. 4. Instantaneous reduced local concentration $X_{T}$.

water feed stream to 2 (white) in the fluorescent tracer feed stream.

More experimental validations are available in Fall, Lecoq, and David (2002).

\section{Model}

The following section is based upon the probabilistic description of mixing by Danckwerts (1952).

Each elementary volume of zone 2 is made of a fraction $f(x, y, z, t)$ of incompressible fluid coming from the feed stream and of the complementary fraction $1-f$, coming from the bulk zone 3 where uniform concentration $C_{T}$ is assumed. In the case described by Fig. $5 \mathrm{a}$ for the feed stream at the left-hand side, where $C_{T}$ is the instantaneous concentration of inert tracer, and $C_{T 0}$ the mixing concentration attained in zone 3 .

And at the right-hand side feed stream (Fig. 5b),

$f_{R}=\left(C_{T}-C_{T 0}\right) /\left(C_{T 0}^{\prime}-C_{T 0}\right)$.

Introducing the flow rate ratio $v=Q_{R} / Q_{L}$,

$f_{R}=v\left(C_{T} / C_{T 0}-1\right)=v\left(X_{T}-1\right)$.

When feeding with reagents $\mathrm{A}$ and $\mathrm{B}$, we assume that uniform concentrations of $\mathrm{A}$ and $\mathrm{B}$ exist in zone 3 and that due to dilution there is no change in hydrodynamics (Figs. 5c and $\mathrm{d}$ ). Then, in zone 2

$$
\begin{aligned}
C_{A R} & =\overline{C_{A}}\left(1-f_{R}\right)+C_{A 0}^{\prime} f_{R}, \\
C_{B R} & =\overline{C_{B}}\left(1-f_{R}\right), \\
C_{A L} & =\overline{C_{A}}\left(1-f_{L}\right), \\
C_{B L} & =\overline{C_{B}}\left(1-f_{L}\right)+C_{B 0}^{\prime} f_{L} .
\end{aligned}
$$




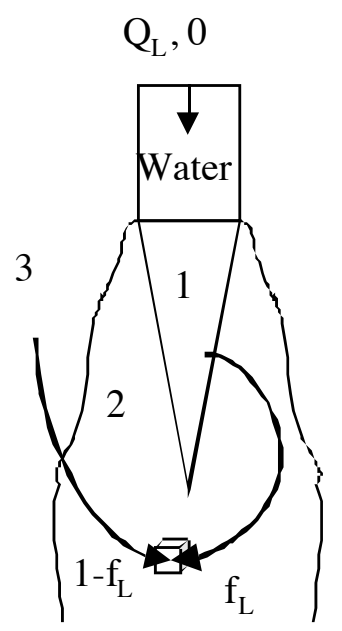

(a)

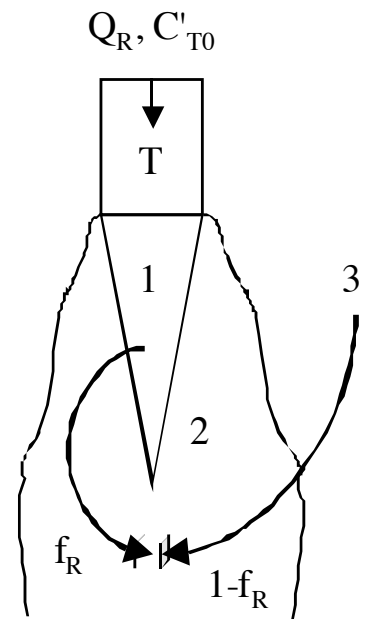

(b)

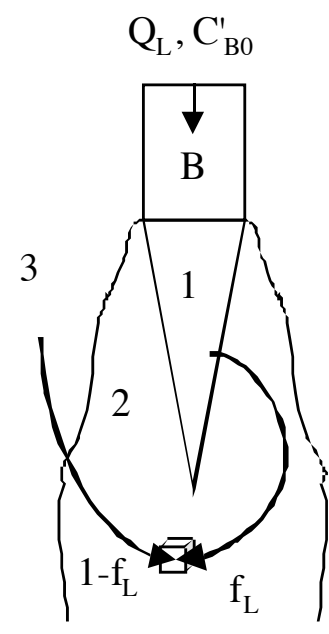

(c)

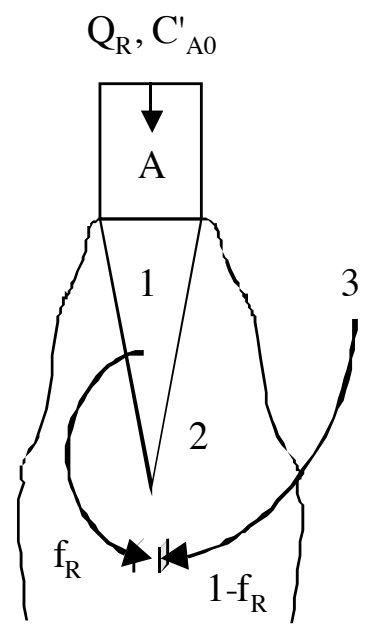

(d)
Fig. 5. The feed stream jets model: (a) Water feed stream; (b) inert tracer feed stream; (c) reagent A feed stream and (d) reagent B feed stream.

We define the relative supersaturation as

$S=\frac{C_{A}^{n} C_{B}^{m}}{P_{S}}$

Then, replacing $C_{A}$ and $C_{B}$ from Eqs. (5) and (6) into Eq. (9), gives in the right feed stream

$$
\begin{aligned}
S_{R}= & \frac{{\overline{C_{B}}}^{m}\left(1-f_{R}\right)^{m}}{P_{S}} \\
& \times\left\{\sum_{i=0}^{n} C_{n}^{i}{\overline{C_{A}}}^{n-i} C^{\prime i}{ }_{A 0}^{i}\left(1-f_{R}\right)^{n-i} f_{R}^{i}\right\} .
\end{aligned}
$$

Keeping in mind that $C_{A 0}^{\prime} \gg \overline{C_{A}}$, except when $f_{R}$ is close to 0 (in that case supersaturation is 1 ), every term of rank $i$ in the summation (10) is larger than the former one by a factor $\frac{(n-i+1) f_{R} C_{A 0}^{\prime}}{i\left(1-f_{R}\right) \overline{C_{A}}}$ and, finally, $S_{R}$ is approximated by keeping only the last term of (10),

$S_{R}=\frac{1}{P_{S}}\left\{C^{\prime n}{ }_{A 0}{\overline{C_{B}}}^{m}\left(1-f_{R}\right)^{m} f_{R}^{n}\right\}$.

An identical derivation can be done for the left feed stream,

$S_{L}=\frac{1}{P_{S}}\left\{C_{B 0}^{\prime m}{\overline{C_{A}}}^{n}\left(1-f_{L}\right)^{n} f_{L}^{m}\right\}$.

Replacing $f_{R}$ and $f_{L}$ by their expressions from Eqs. (2) and (4), and averaging,

$\overline{S_{R}}=\frac{1}{P_{S}}\left\{C^{\prime n}{ }_{A 0}{\overline{C_{B}}}^{m} \overline{\left(1+v-v X_{T}\right)^{m} v^{n}\left(X_{T}-1\right)^{n}}\right\}$,

$\overline{S_{L}}=\frac{1}{P_{S}}\left\{C_{B 0}^{\prime m}{\overline{C_{A}}}^{n} \overline{\left(1-X_{T}\right)^{m}\left(X_{T}\right)^{n}}\right\}$.

If we consider now that the feed is stoichiometric and assuming to be close to equilibrium in the zone 3 , which is all the more reasonable as the tank residence time is large compared to the jet residence time,

$n \overline{C_{A}}=m \overline{C_{B}}$,

${\overline{C_{A}}}^{n}{\overline{C_{B}}}^{m} \approx P_{S}$,

which yields

$\overline{C_{A}}=P_{s}^{1 /(n+m)}\left(\frac{m}{n}\right)^{m /(m+n)}$,

$\overline{C_{B}}=P_{s}^{1 /(n+m)}\left(\frac{n}{m}\right)^{n /(m+n)}$.

In that situation, Eqs. (14) and (15) become

$\overline{S_{R}}=\frac{1}{P_{S}^{n /(n+m)}}$

$$
\times\left\{C^{\prime n}{ }_{A 0}\left(\frac{n}{m}\right)^{n m /(n+m)} \overline{\left(1+v-v X_{T}\right)^{m} v^{n}\left(X_{T}-1\right)^{n}}\right\},
$$

$\overline{S_{L}}=\frac{1}{P_{S}^{m /(n+m)}}\left\{C_{B 0}^{\prime m}\left(\frac{m}{n}\right)^{n m /(n+m)} \overline{\left(1-X_{T}\right)^{m}\left(X_{T}\right)^{n}}\right\}$.

Expressions (18) and (19) simplify if $n=m=1$

$\overline{S_{R}}=\frac{1}{P_{S}^{1 / 2}}\left\{C_{A 0}^{\prime} v\left((2 v+1) \overline{X_{T}}-1-v-v \overline{X_{T}^{2}}\right)\right\}$,

$\overline{S_{L}}=\frac{1}{P_{S}^{1 / 2}}\left\{C_{B 0}^{\prime}\left(\overline{X_{T}}-\overline{X_{T}^{2}}\right)\right\}$.

Note that the average supersaturation is nil when $X_{T}=1+1 / v$ (right jet), $X_{T}=0$ (left jet), or $X_{T}=1$ (both jets).

The next Fig. 6 shows the averaged supersaturation field $\overline{S_{R}} / S_{0}^{\prime}$ for the mixing device of Figs. 2-3, calculated from the instantaneous concentration fields for the case $n=m=1$ and $v=1$ and with $S_{0}^{\prime}=C_{A 0}^{\prime} / \sqrt{P_{s}}$.

The next step consists of the calculation the nucleation fluxes. Nucleation rate, as well homogeneous as heterogeneous nucleation, is generally expressed by the Volmer and 


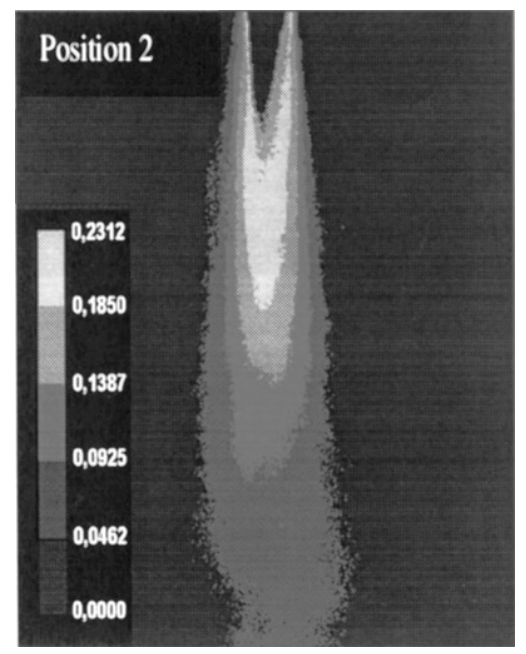

Fig. 6. Mean supersaturation field in the feed stream containing fluorescent tracer, $\overline{S_{R}} / S_{0}^{\prime}$.

Weber (1926) relationship

$R_{N}=A_{N} \exp \left(\frac{-B_{N}}{\log _{e}^{2} S}\right)$

$B_{N}$ values between 1 and 50 denote primary heterogeneous nucleation, whereas values between 50 and 1000 are generally attributed to homogeneous nucleation (Kashiev, 2000).

Integrating the expressions (18) and (19) of S into relation (22), one yields the local instantaneous nucleation rate. The following expressions are obtained for the right and left averaged nucleation rates: $v=1$ in Figs. $7 \mathrm{a}-\mathrm{f}$. The patterns are similar to Fig. 6 for average supersaturation, but the intensity of $\overline{R_{N R}} / A_{N}$ strongly depends on $S_{0}^{\prime}$ and $B_{N}$ values. The nucleation region spreads out at constant $S_{0}^{\prime}$ while $B_{N}$ diminishes. However, this does not mean at all that the total nucleation rate is enhanced by heterogeneous nucleation, because much higher values of $A_{N}$ prevail for homogeneous nucleation (Mersmann, 2001).

Small values of $B_{N}$ correspond to heterogeneous primary nucleation whereas high values correspond to homogeneous primary nucleation. For the lower values of $B_{N}$, there is no change in size and intensity of the nucleation source with increasing initial supersaturation $S_{0}^{\prime}$.

Conversely, increasing $S_{0}^{\prime}$ strongly influences the nucleation flux at high $B_{N}$ values, creating an ignition-like phenomenon.

Finally, taking literature (Dirksen \& Ring, 1991) values of $A_{N}$ and $B_{N}$ for barium sulphate precipitation, we have checked the well-known switch over effect from predominant heterogeneous to predominant homogeneous nucleation due to a concentration increase. This is verified in Figs. 8a-f.

Note that the zone of nucleation flux spreads out much wider for heterogeneous nucleation (Fig. 8b, resp. Fig. 8e) than for homogeneous nucleation (Fig. 8a, resp. Fig. 8d). Moreover, for large values of initial supersaturation $S_{0}^{\prime}$ (Fig. 8d compared to Fig. 8e), the flux values are locally higher for homogeneous nucleation but concentrated on a smaller area.

Furthermore, when comparing predominant heterogeneous situation (Fig. 8c) with the predominant homogeneous one (Fig. 8f) the values of $\int_{\text {image }} \overline{R_{N R}} \mathrm{~d} x \mathrm{~d} y$ are
$\overline{R_{N R}}=A_{N} \exp \left(\frac{-B_{N}}{\log _{e}^{2}\left\{\left(1 / P_{s}^{n /(n+m)}\right) C_{A 0}^{\prime n}(n / m)^{n m /(n+m)} v^{n}\left(1+v-v X_{T}\right)^{m}\left(X_{T}-1\right)^{n}\right\}}\right)$,

$\overline{R_{N L}}=A_{N} \exp \left(\frac{-B_{N}}{\log _{e}^{2}\left\{\left(1 / P_{s}^{m /(n+m)}\right) C_{B 0}^{\prime m}(m / n)^{n m /(n+m)}\left(1-X_{T}\right)^{m}\left(X_{T}\right)^{n}\right\}}\right)$.
Another way to calculate these nucleation rates is to make use of the experimental probability density function (PDF) $p\left(X_{T}\right)$ of the reduced concentration of tracer $X_{T}$. For instance, in the simplified case $n=m=1$ and $v=1$
$2.7 \times 10^{24}$ and $3.8 \times 10^{28} \mathrm{~m}^{-1} \mathrm{~s}^{-1}$, respectively. The predominant homogeneous nucleation thus yields higher fluxes at high supersaturations.

$\overline{R_{N R}}=A_{N} \int_{X_{T}=0}^{2} \exp \left(\frac{-B_{N}}{\log _{e}^{2}\left\{\left(C_{A 0}^{\prime} / P_{S}^{1 / 2}\right)\left(2-X_{T}\right)\left(X_{T}-1\right)\right\}}\right) p\left(X_{T}\right) \mathrm{d} X_{T}$,

$\overline{R_{N L}}=A_{N} \int_{X_{T}=0}^{2} \exp \left(\frac{-B_{N}}{\log _{e}^{2}\left\{\left(C_{B 0}^{\prime} / P_{S}^{1 / 2}\right)\left(1-X_{T}\right)\left(X_{T}\right)\right\}}\right) p\left(X_{T}\right) \mathrm{d} X_{T}$.

Six maps of reduced $\overline{R_{N R}} / A_{N}$ are shown for different couples of values of $B_{N}$ and $S_{0}^{\prime}=C_{A 0}^{\prime} / \sqrt{P_{S}}$ with $n=m=1$ and 

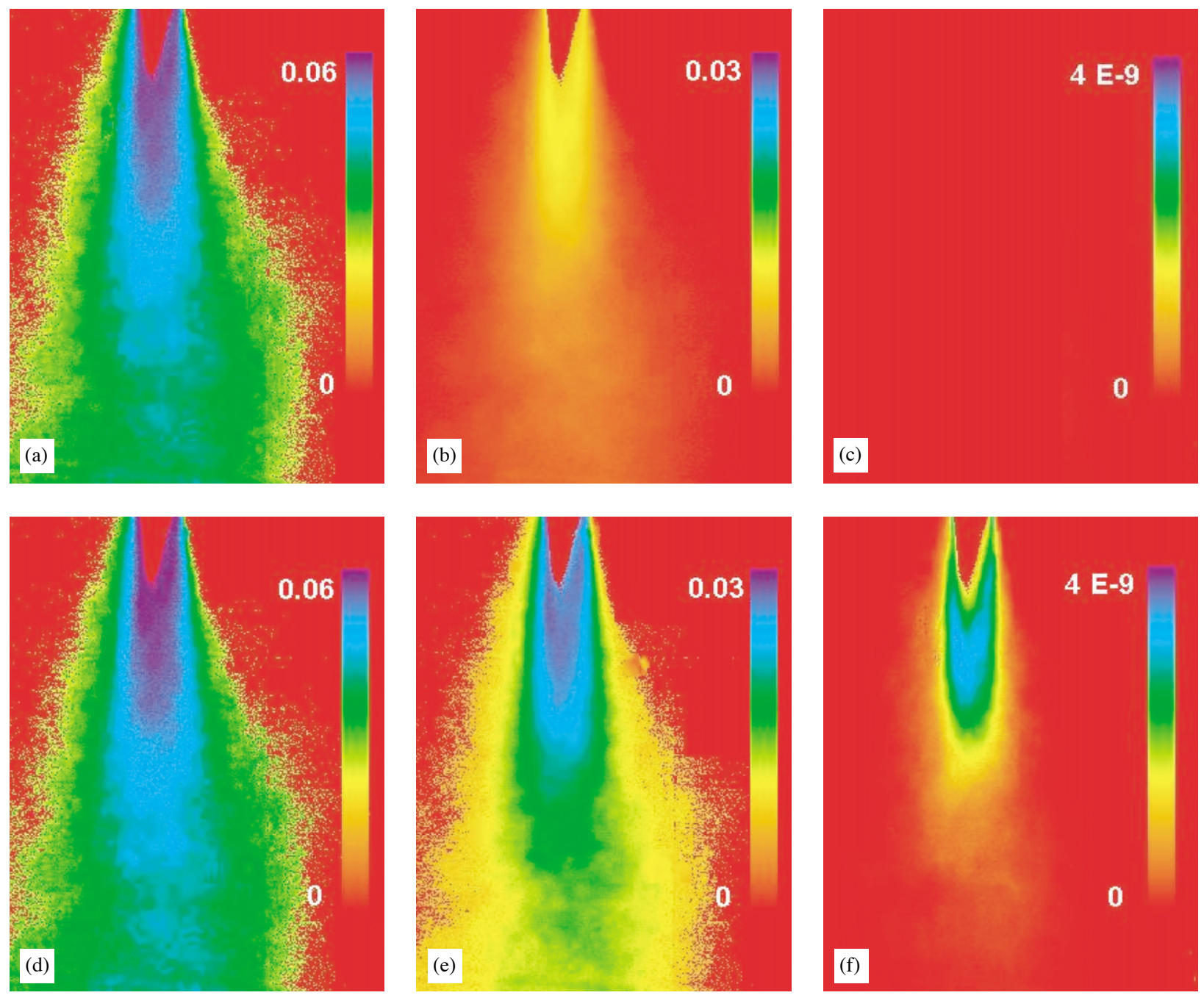

Fig. 7. Reduced nucleation rates $\bar{R}_{N R} / A_{N}$ with: (a) $B_{N}=1$ and $S_{0}^{\prime}=1000$; (b) $B_{N}=50$ and $S_{0}^{\prime}=1000$; (c) $B_{N}=1000$ and $S_{0}^{\prime}=1000$; (d) $B_{N}=1$ and $S_{0}^{\prime}=10000 ;$ (e) $B_{N}=50$ and $S_{0}^{\prime}=10000$ and (f) $B_{N}=1000$ and $S_{0}^{\prime}=10000$.

\section{Case of partially premixed feed streams}

One may try to implement the procedure described above in the case of partially premixed feed streams. However, an additional difficulty arises when doing so. Imagine the situation depicted by Fig. 9, where the mixing zone of both fresh fluid feed streams is described by two fractions $f_{R}$ and $f_{L}$, which denote the fluid fractions at point $(x, y, z)$ and time $t$ which originate from feed tubes $R$ and $L$, respectively.

$1-X_{T}=f_{L}-f_{R} / v$.

A large number of combinations of $f_{R}$ and $f_{L}$ yield the same value of $X_{T}$, except for either $X_{T}=1+1 / v\left(f_{R}=1\right.$ and $\left.f_{L}=0\right)$ or $X_{T}=0\left(f_{R}=0\right.$ and $\left.f_{L}=1\right)$. In the mixing region $C_{A}$ and $C_{B}$ can be written in the same manner as in the unmixed case:

$$
\begin{aligned}
C_{A} & =\overline{C_{A}}\left(1-f_{R}-f_{L}\right)+C_{A 0}^{\prime} f_{R}, \\
C_{B} & =\overline{C_{B}}\left(1-f_{R}-f_{L}\right)+C_{B 0}^{\prime} f_{L} .
\end{aligned}
$$

Thus, from Eq. (9), we have an infinite number of values of supersaturations corresponding to one $X_{T}$ value. In other words, a pixel showing the reduced tracer concentration $X_{T}$ may be fed from different combinations of the bulk, the tracer solution feed and the pure solvent feed, but these combinations will result in different supersaturation values. The only way for overcoming that difficulty is to introduce a mixing model. This will be done in a further paper.

\section{Discussion}

Let us verify if our assumption about the negligible consumption of reagents in the mixing zone 2 holds. The local mass flux in zone 2, which is transferred from the solution by the growing crystal, writes

$$
\begin{aligned}
F= & 3 \rho \Phi_{v} \int_{V_{2}} \int_{0}^{\infty} G(x, y, z, L) L^{2} \Psi(x, y, z, L) \\
& \times \mathrm{d} L \mathrm{~d} x \mathrm{~d} y \mathrm{~d} z .
\end{aligned}
$$



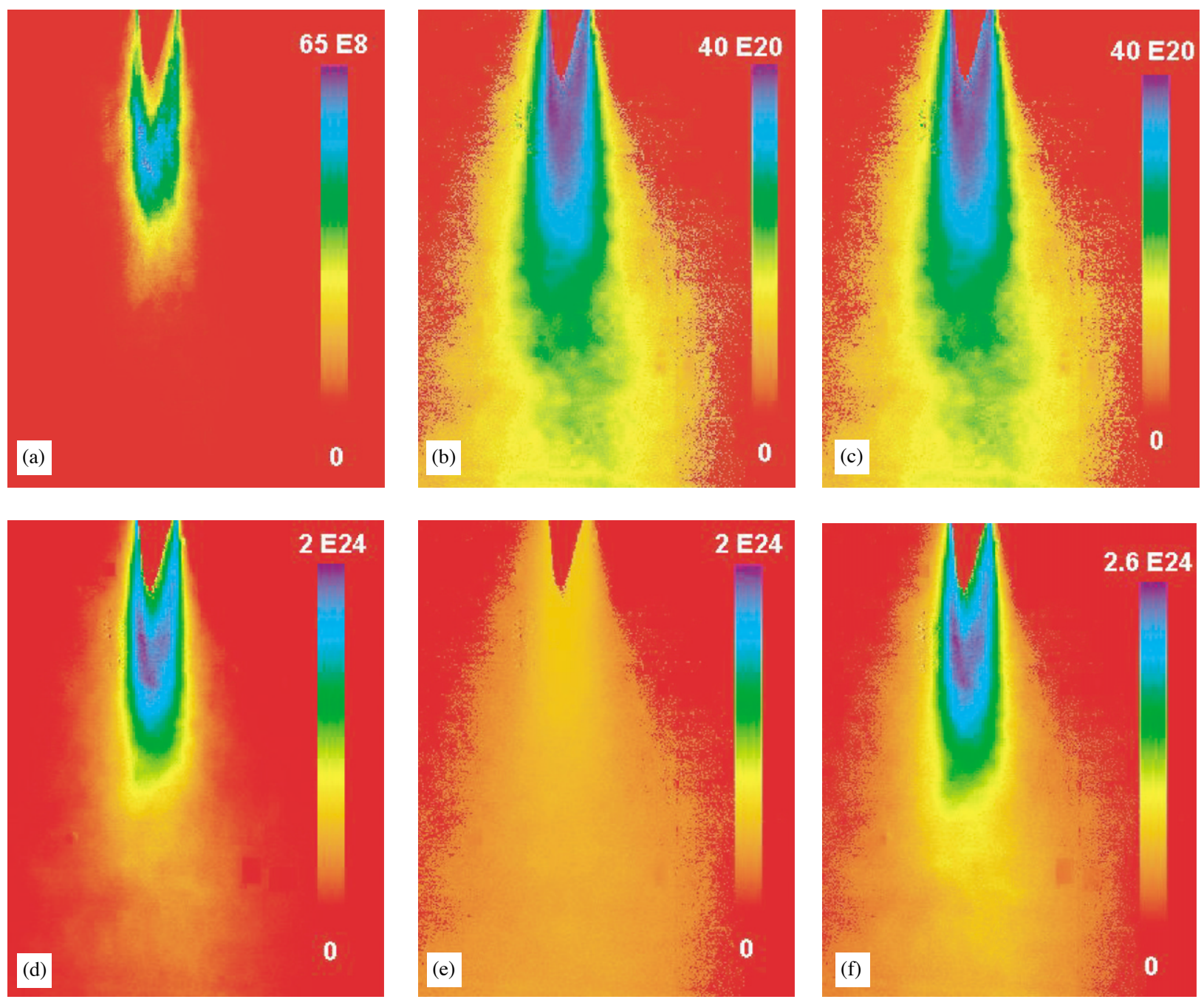

Fig. 8. (a) Nucleation rate $\bar{R}_{N R}$ in homogeneous case with $\left(S_{0}^{\prime}=300 ; A_{N}=10^{30} ; B_{N}=800\right)$. (b) Nucleation rate $\bar{R}_{N R}$ in heterogeneous case with $\left(S_{0}^{\prime}=300 ; A_{N}=10^{25} ; B_{N}=8\right)$. (c) Overall nucleation rate $\bar{R}_{N R}$, sum of both nucleation rates of Fig. $8 \mathrm{a}\left(S_{0}^{\prime}=300 ; A_{N}=10^{30} ; B_{N}=800\right)$ and Fig. $8 \mathrm{~b}\left(S_{0}^{\prime}=300 ; A_{N}=10^{25} ; B_{N}=8\right)$. (d) Nucleation rate $\bar{R}_{N R}$ in homogeneous case with $\left(S_{0}^{\prime}=30000 ; A_{N}=10^{30} ; B_{N}=800\right)$. (e) Nucleation rate $\bar{R}_{N R}$ in heterogeneous case with $\left(S_{0}^{\prime}=30000 ; A_{N}=10^{25} ; B_{N}=8\right)$. (f) Overall nucleation rate $\bar{R}_{N R}$, sum of both nucleation rates of Fig. 8d ( $S_{0}^{\prime}=30000$; $\left.A_{N}=10^{30} ; B_{N}=800\right)$ and Fig. $8 \mathrm{e}\left(S_{0}^{\prime}=30000 ; A_{N}=10^{25} ; B_{N}=8\right)$.

One may compare this flux with the inlet flux of fresh reagents $\mathrm{A}$ or $\mathrm{B}$, which are in close relationship together because of the stoichiometric feed.

$\eta=\frac{3 \rho n \Phi_{v} \int_{V_{2}} \int_{0}^{\infty} G(x, y, z, L) L^{2} \Psi(x, y, z, L) \mathrm{d} L \mathrm{~d} x \mathrm{~d} y \mathrm{~d} z}{M Q_{R} C_{A 0}^{\prime}}$.

If we assume uniform particle size distribution and growth rate throughout the tank it is possible to grossly evaluate the maximum value of $\eta$. The instantaneous conversion flux from the liquid to the solid phase in the whole tank will be constrained by the inlet flux of A:

$$
\begin{gathered}
3 \rho n \Phi_{v} V_{T} \int_{0}^{\infty} G L^{2} \Psi(L) \mathrm{d} L \approx 3 \rho n \Phi_{v} V_{T} \\
\times G \int_{0}^{\infty} L^{2} \Psi(L) \mathrm{d} L \leqslant M Q_{R} C_{A 0}^{\prime} .
\end{gathered}
$$

Consequently, $\eta \leqslant V_{2} / V_{T}$, which is very small. Of course, one may estimate that $\Psi(L)$ is much higher in the zone 2 due to enhanced nucleation, but these nuclei are so small that $L^{2} \Psi(L)$ remains limited. Moreover, variations of $G$ are limited for the larger crystals (with higher weight in Eq. (29)) by the upper diffusional boundary. Taking typical values of parameters and variables in Eq. (30) $\left(\rho=2000 \mathrm{~kg} \mathrm{~m}^{-3} ; \Phi_{v}=0.5 ; n=1 ; V_{2}=2.5 \times 10^{-5} \mathrm{~m}^{3} ;\right.$ $L=10^{-8} \mathrm{~m} ; M=0.1 \mathrm{~kg} \mathrm{~mol}^{-1} ; C_{A 0}^{\prime}=10 \mathrm{~mol} \mathrm{~m}^{-3}$ and, according to Mersmann (2001), high values of $\int_{0}^{\infty} \Psi(L) \mathrm{d} L=10^{18} \mathrm{~m}^{-3}$ and constant $G=10^{-7} \mathrm{~m} / \mathrm{s}$, one obtains $\eta=2.5 \%$.

The conclusion is that neither the average growth of existing crystals nor a high nucleation rate is able to seriously deplete the reagent's concentrations in the mixing zone 2 .

Coming back to Eqs. (20) and (21) in the case $v=1$, the variance $\sigma_{T}^{2}$ of the reduced tracer concentration may be 


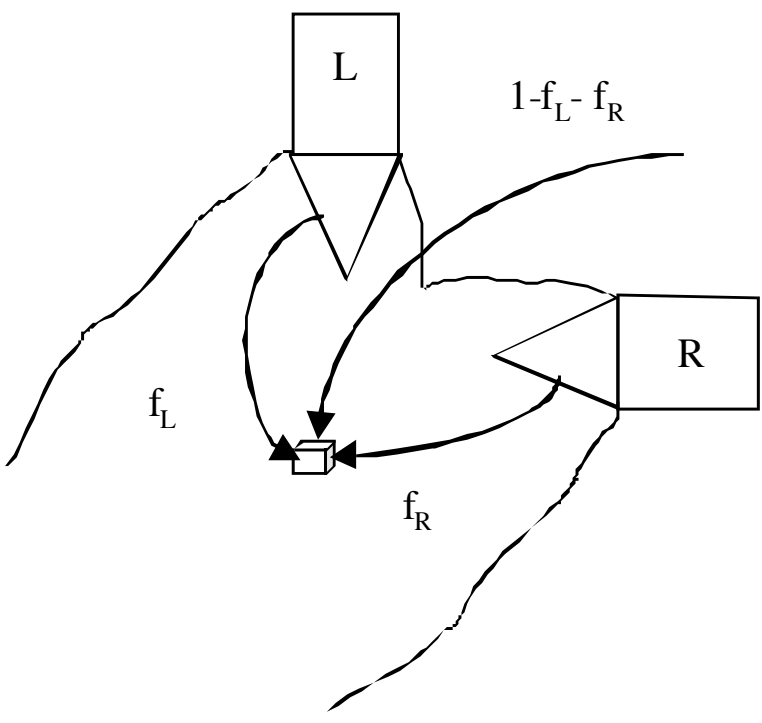

Fig. 9. Partially premixed feed streams model.

introduced as follows:

$\overline{S_{R}}=\frac{C_{A 0}^{\prime}}{P_{S}^{1 / 2}}\left(3 \overline{X_{T}}-2-\sigma_{T}^{2}-{\overline{X_{T}}}^{2}\right)$,

$\overline{S_{L}}=\frac{C_{B 0}^{\prime}}{P_{S}^{1 / 2}}\left(\overline{X_{T}}-\sigma_{T}^{2}-{\overline{X_{T}}}^{2}\right)$.

The paradox is that the right and left average supersaturations are decreasing functions of the variance $\sigma_{T}^{2}$. This could seem contradictory with the experimental findings of Houcine, Plasari, David, and Villermaux (1997). These authors had observed an obvious correlation between large variance zones of tracer fluctuations around the feed streams and the high nucleation rates when the reagents were fed at the same location.

Eqs. (32) indicate that the highest average supersaturations are obtained at locations where (a) $\overline{X_{T}} \neq 0,1$, or 2 , and (b) $\sigma_{T}^{2}$ is small (i.e. reduced fluctuations of tracer concentration). Indeed, these areas are the same as those for non-zero variance, but the locations of maximum supersaturations are different from those for maximum variance.

Other points of discussion are the spatial and temporal resolution of the PLIF.

If an observation system integrates the concentration signal over a too large time interval, fluctuations at high frequencies are not monitored. The illumination time of every pixel is about $35 \mathrm{~ms}$ for our experiments.

The spatial resolution of PLIF is about $100 \mu \mathrm{m}$ in our example. By focusing the camera on a smaller frame, it can be lowered down to a $10 \times 10 \times 100 \mu \mathrm{m}$ volume. This makes the observation scale comparable to the Kolmogorov length scale in a stirred tank. It means that the larger structures where kinetic energy is dissipated are taken into account. However, the eddy laminar stretching and swirling step, whose size is beyond the Kolmogorov scale down to the Batchelor scale, i.e. a few $\mu \mathrm{m}$, is not observable by these means. This stretching and swirling process is of major importance as it governs the extent and selectivity of chemical reactions (Baldyga \& Bourne, 1999). For non-viscous aqueous solutions, the subsequent diffusion step is so short that it can be neglected.

Therefore, a description in terms of micromixing of the stretching and swirling process is mandatory if one wants to precisely calculate the supersaturation and the nucleation flux inside the pixels. This modeling is, however, simplified by the fact that one may neglect chemical consumption of the reagents by both crystal growth and nucleation, as demonstrated above. Additionally, stretching and swirling is rapid when compared with residence times in the inlet jet, so that it may be considered as a local process, depending only on the local energy dissipation rate in the pixel's volume. Thus, the calculated supersaturation fields of the present paper should be considered as first approximations. Also, in a more developed model, other processes like agglomeration and secondary nucleation could be taken into account.

\section{Conclusion}

In the case of unmixed feed streams in a precipitator it has been shown that it is likely to derive a supersaturation map and a nucleation flux from the knowledge of a concentration field of an inert tracer in the same device. There is a strong similarity between the images of average supersaturation and those of the nucleation fluxes.

The assumption of negligible consumption of reagents in the mixing jets has been verified. But, the method remains limited by the three-dimensional spatial resolution of the experimental image of inert tracer concentration.

It is now possible, with a good approximation, to qualify a mixer and the associate feed locations and geometry from the point of view of their ability to generate a high or low nucleation flux for a given precipitation from a simple tracer observation. The choice of the best suitable mixing situation for every purpose is thus facilitated.

The extension of the present work to the case of premixed feed streams is somewhat more difficult and will be tackled in a further paper.

\section{Notation}

$A_{N} \quad$ constant, Eq. (22), $\mathrm{m}^{-3} \mathrm{~s}^{-1}$

$B_{N} \quad$ constant, Eq. (22), dimensionless

$C$ concentration, $\mathrm{mol} \mathrm{m} \mathrm{m}^{-3}$

$\overline{C_{A}}$ mean mixing concentration of reagent $\mathrm{A}$ in the outlet, $\mathrm{mol} \mathrm{m}^{-3}$

$\frac{C_{A 0}^{\prime}}{C_{B}}$ feed concentration of reagent $\mathrm{A}, \mathrm{mol} \mathrm{m}^{-3}$

$\overline{C_{B}}$ mean mixing concentration of reagent $\mathrm{B}$ in the outlet, $\mathrm{mol} \mathrm{m}^{-3}$

$C_{B 0}^{\prime}$ feed concentration of reagent $\mathrm{B}, \mathrm{mol} \mathrm{m}^{-3}$ 
$C_{T 0}$ mixing tracer concentration in the outlet, $\mathrm{mol} \mathrm{m}^{-3}$

$C_{T 0}^{\prime}$ feed concentration of fluorescent tracer, $\mathrm{mol} \mathrm{m}^{-3}$

$f_{L}$ feed stream fraction coming from the left inlet, dimensionless

$f_{R} \quad$ feed stream fraction coming from the right inlet, dimensionless

$G \quad$ linear growth rate, $\mathrm{m} \mathrm{s}^{-1}$

$H \quad$ height of the liquid in the tank, $\mathrm{m}$

$L \quad$ crystal size, $\mathrm{m}$

$M \quad$ molar mass, $\mathrm{kg} \mathrm{mol}^{-1}$

$N \quad$ stirring speed, $\mathrm{s}^{-1}$

$P_{S} \quad$ solubility product, $\mathrm{mol}^{2} \mathrm{~m}^{-6}$

$Q \quad$ feed rate, $\mathrm{dm}^{3} \mathrm{~s}^{-1}$

$R_{N} \quad$ nucleation flux, $\mathrm{m}^{-3} \mathrm{~s}^{-1}$

$S \quad$ supersaturation, dimensionless

$S_{0}^{\prime} \quad$ initial local supersaturation, $S_{0}^{\prime}=C_{A 0}^{\prime} / \sqrt{P_{S}}$, dimensionless

$T \quad$ internal diameter of the tank, $\mathrm{m}$

$v \quad$ flow rate ratio, dimensionless

$V \quad$ volume, $\mathrm{m}^{3}$

$X_{T} \quad$ reduced local concentration of the tracer, dimensionless

\section{Greek letters}

$\eta \quad$ ratio growth to inlet flux, dimensionless

$\Phi_{v} \quad$ volumic shape factor, dimensionless

$\Psi \quad$ number distribution density, $\mathrm{m}^{-4}$

$\rho \quad$ solid density, $\mathrm{kg} \mathrm{m}^{3}$

\section{Subscripts}

$\begin{array}{ll}A & \text { reactant A } \\ B & \text { reactant B } \\ L & \text { left } \\ R & \text { right } \\ T & \text { fluorescent tracer }\end{array}$

\section{Superscripts}

$m, n \quad$ stoichiometric coefficients, dimensionless

- time average, dimensionless

\section{References}

Baldyga, J., \& Bourne, J. R. (1999). Turbulent mixing and chemical reactions (810-840). Chichester, New York, Weinheim, Brisbane, Singapore, Toronto: Wiley.

Danckwerts, P. V. (1952). The definition and measurement of some characteristics of mixtures. Applied Scientific Research Section A, 3, 279-296.

Dirksen, J. A., \& Ring, T. A. (1991). Fundamentals of crystallization: Kinetic effects on particle size distributions and morphology. Chemical Engineering Science, 46(10), 2389-2427.

Fall, A., Lecoq, O., \& David, R. (2001). Characterization of mixing in a stirred tank by planar laser induced fluorescent (PLIF). Trans IChemE, 79(A), 876-882.

Fall, A., Lecoq, O., \& David, R. (2002). Derivation of supersaturation and nucleation flux in a stirred tank from tracer concentration measurements by PLIF. 15th international symposium on industrial crystallization, Sorrento, Italy.

Houcine, I., Plasari, E., David, R., \& Villermaux, J. (1997). Influence of mixing characteristics on the quality and size of precipitated calcium oxalate in a pilot scale reactor. Trans IChemE, 75(Part A), 252-256.

Houcine, I., Vivier, H., Plasari, E., David, R., \& Villermaux, J. (1996). Planar laser induced fluorescence technique for measurements of concentration fields in continuous stirred tank reactors. Experiments in Fluids, 22, 95-102.

Kashiev, D. (2000). Nucleation: Basic theory with applications (9-69). Oxford, Auckland Boston, Johannesburg, Melbourne, New Delhi: Butterworth-Heinemann.

Levenspiel, O. (1971). Chemical reaction engineering: Mixing of fluids. New-York: Wiley.

Mersmann, A. (2001). Crystallization technology handbook (2nd ed.) (pp. 45-79). New York: Marcel Dekker.

Villermaux, J., \& David, R. (1988). Effet du micromélange sur la précipitation. Journal de Chimie Physique, 85, 273-279.

Volmer, M., \& Weber, A. (1926). Keimbildung in übersättigten Gebilden. Zeitschirft fuer Physikalische Chemie, 119, 277-301.

Shenoy, U. V., \& Toor, H. L. (1990). Unifying indicator and instantaneous reaction methods of measuring micromixing. AIChE Journal, 36(2), $227-232$. 